\title{
A DOMAIN DECOMPOSITION APPROACH FOR NON-CONFORMAL COUPLINGS BETWEEN FINITE AND BOUNDARY ELEMENTS FOR ELECTROMAGNETIC SCATTERING PROBLEMS in $\mathcal{R}^{3}$
}

\author{
Marinos Vouvakis and Jin-Fa Lee \\ ElectroScience Lab., Electrical and Computer Engineering Department, Ohio State \\ University, 1320 Kinnear Rd., Columbus, OH 43212, USA \\ vouvakis.1@osu.edu, lee.1863@osu.edu
}

\section{Introduction}

To solve electromagetic scattering problems in $\mathcal{R}^{3}$, the popular approach is to combine and couple the finite and boundary elements. Common engineering practises in coupling the finite and boundary elements usually result in nonsymmetric and non-variational formulations [1, 2]. The symmetric coupling between finite and boundary elements was first proposed by Costabel [3] in 1987. Since then, quite a few papers being published on the topic of symmetric couplings. Among them, we list references $[4,5,6,7]$. In particular, references $[5,6,7]$ dealt with variational formulations for solving electromagnetic wave radiation and scattering problems. Although the formulations detailed in $[5,6$, 7] result in symmetric couplings between finite and boundary elements, they still suffer the notorious internal resonances. The purpose of this chapter is to present a variational formulation, which couples finite and boundary elements through non-conformal meshes. The formulation results in matrix equations that are symmetric, coercive, and free of internal resonances.

Our plan for this chapter is as follows. Section 2 details the proposed variational formulation for non-conformal couplings between finite and boundary elements. In section 3, we show that, through a box computational domain, the proposed formulation is free of internal resonances and it satisfies the C.B.S inequality [8]. Moreover, in section 3 we validate the accuracy of the proposed formulation by a complex scattering problem. A brief conclusion is provided in section 4 . 


\section{Formulation}

\subsection{Boundary Value Problems}

This chapter considers the solution of an electromagnetic scattering problem in $\mathcal{R}^{3}$. A finite computational domain, $\Omega \subset \mathcal{R}^{3}$, encloses all the scatterers inside. The exterior region, $\Omega^{c}=\mathcal{R}^{3} / \bar{\Omega}$, is then homogeneous and assumed to be free space. Let $\mathbf{E}$ denotes the scattered electric field in the exterior region $\Omega^{c}$ and the total electric field inside $\Omega$. It is then the solution of the transmission problem [5]:

$$
\begin{aligned}
& \nabla \times \nabla \times \mathbf{E}-k^{2} \mathbf{E}=\quad 0 \quad \text { in } \Omega^{c} \\
& \nabla \times \frac{1}{\mu_{r}} \nabla \times \mathbf{E}-k^{2} \epsilon_{r} \mathbf{E}=0 \quad \text { in } \Omega \\
& {\left[\gamma_{t} \mathbf{E}\right]_{\Gamma}=\gamma_{t} \mathbf{E}^{i n c},\left[\frac{1}{\mu_{r}} \gamma_{N} \mathbf{E}\right]_{\Gamma}=\gamma_{N} \mathbf{E}^{i n c} \text { on } \Gamma} \\
& \lim _{|\mathbf{X}| \rightarrow \infty} \nabla \times \mathbf{E} \times \mathbf{x}-i k|\mathbf{x}| \mathbf{E}=\quad 0
\end{aligned}
$$

In Eq. 1, $k$ is the wavenumber in free space, the two surface trace operators are $\gamma_{t} \mathbf{E}=\mathbf{n} \times \mathbf{E} \times \mathbf{n}$ for the tangential components of $\mathbf{E}$ on $\Gamma$ and $\gamma_{N} \mathbf{E}=\nabla \times \mathbf{E} \times \mathbf{n}$ for the "magnetic trace" on $\Gamma$. The surface unit normal $\mathbf{n}$ points from $\Omega$ toward the exterior region $\Omega^{c}$. Finally, $[\gamma \phi]_{\Gamma}=\left.\gamma \phi\right|_{\Omega}-\left.\gamma \phi\right|_{\Omega^{c}}$ denotes the jump of a function $\phi$ across $\Gamma$.

The current formulation starts first by introducing two "cement" variables $[9], \mathbf{j}^{-}$and $\mathbf{j}^{+}$, on the boundary $\Gamma$. These two cement variables related to the electric currents on $\Gamma$ in $\Omega$ and $\Omega^{c}$, respectively. Subsequently, the original transmission problem Eq. 1 can be stated alternatively as:

$$
\begin{array}{r}
\text { in } \Omega \\
\nabla \times \frac{1}{\mu_{r}} \nabla \times \mathbf{E}-k^{2} \epsilon_{r} \mathbf{E}=0 \\
\frac{1}{\mu_{r}} \gamma_{N} \mathbf{E}=\mathbf{j}^{-} \\
\text {in } \Omega^{c} \\
\nabla \times \nabla \times \mathbf{E}=0 \\
\lim _{|\mathbf{x}| \rightarrow \infty} \nabla \times \mathbf{E} \times \mathbf{x}-i k|\mathbf{x}| \mathbf{E}=0 \\
-\gamma_{N} \mathbf{E}=\mathbf{j}^{+}
\end{array}
$$

Transmission Conditions on $\Gamma$

$$
\begin{aligned}
& \mathbf{e}^{-}-\mathbf{e}^{+}=\gamma_{t} \mathbf{E}^{i n c} \\
& \mathbf{j}^{-}+\mathbf{j}^{+}=\gamma_{N} \mathbf{E}^{i n c}
\end{aligned}
$$

However, direct numerical implementation based on the transmission conditions 4 is not desirable since they are closely related to the Dirichlet and 
Neumann mappings, which usually subject the sub-domains to the "internal resonances" during the solution process. Taking our que from the domain decomposition literature, we simply replace 4 by Robin transmission conditions [9]. Namely,

$$
\begin{array}{r}
\text { Robin Transmission Conditions on } \Gamma \\
-i k \mathbf{e}^{-}+\eta \mathbf{j}^{-}=-i k \mathbf{e}^{+}-\eta \mathbf{j}^{+}-\mathbf{f}^{i n c} \\
-i k \mathbf{e}^{+}+\eta \mathbf{j}^{+}=-i k \mathbf{e}^{-}-\eta \mathbf{j}^{-}+\mathbf{g}^{i n c}
\end{array}
$$

where $\mathbf{f}^{i n c}=i k \gamma_{t} \mathbf{E}^{i n c}+\gamma_{N} \mathbf{E}^{i n c}$ and $\mathbf{g}^{i n c}=i k \gamma_{t} \mathbf{E}^{i n c}-\gamma_{N} \mathbf{E}^{i n c}$.

\subsection{Galerkin Variational Formulation}

From the physical consideration that both the electric and magnetic energies of the system need be finite, it is transparent to see that the vector field $\mathbf{E}$ in Eq. 1 resides in the product space $\mathbf{H}(\operatorname{curl} ; \Omega) \times \mathbf{H}_{l o c}\left(\operatorname{curl} ; \Omega^{c}\right)$ [5]. To establish the proper spaces of the tangential traces $\mathbf{e}^{-}, \mathbf{e}^{+}$as well as the cement variables $\mathbf{j}^{-}$and $\mathbf{j}^{+}$, we borrow heavily from [5] the following results:

Theorem 1. The trace mappings $\gamma_{t}^{+}: \boldsymbol{H}_{l o c}\left(\boldsymbol{c u r l} ; \Omega^{c}\right) \mapsto \boldsymbol{H}^{-1 / 2}\left(\operatorname{curl}_{\Gamma}, \Gamma^{+}\right)$, $\gamma_{t}^{-}: \boldsymbol{H}(\boldsymbol{c u r l} ; \Omega) \mapsto \boldsymbol{H}^{-1 / 2}\left(\right.$ curl $\left._{\Gamma}, \Gamma^{-}\right)$are continuous and surjective. Moreover, the traces $\gamma_{N}^{ \pm}$furnish continuous mappings: $\gamma_{N}^{+}: \boldsymbol{H}_{l o c}\left(\boldsymbol{c u r l}^{2} ; \Omega^{c}\right) \mapsto$ $\boldsymbol{H}^{-1 / 2}\left(\operatorname{div}_{\Gamma}, \Gamma^{+}\right)$and $\gamma_{N}^{-}: \boldsymbol{H}\left(\operatorname{curl}^{2} ; \Omega\right) \mapsto \boldsymbol{H}^{-1 / 2}\left(\operatorname{div}_{\Gamma}, \Gamma^{-}\right)$.

Now we are ready to state the variational formulation which couples the finite and boundary elements on non-conformal meshes. By non-conformity, we refer to the fact that the triangulation on $\Gamma^{-}$need not be the same as the triangulation on $\Gamma^{+}$. This non-conformity feature admits two major benefits: (a) Different orders of polynomial approximations can be employed separately for finite elements and boundary elements. Subsequently, the triangulations on $\Gamma^{-}$and $\Gamma^{+}$would require drastically different spatial resolutions; and, (b) In the process of goal-oriented adaptive mesh refinements [10], the triangulation on $\Gamma^{-}$often become un-necessary fine in certain regions for the boundary elements. The non-conformal coupling approach allows for a more uniform triangulation on $\Gamma^{+}$and hence can greatly reduce the computational burden.

In $\Omega$, the variational formulation for the finite elements can be stated as

$$
\text { Seek } \mathbf{E} \in \mathbf{H}(\operatorname{curl} ; \Omega) \text { such that }
$$

$$
\begin{gathered}
a(\mathbf{v}, \mathbf{E})-\left\langle\gamma_{t} \mathbf{v}, \mathbf{j}^{-}\right\rangle_{\Gamma^{-}}=0 \\
\forall \mathbf{v} \in \mathbf{H}(\mathbf{c u r l} ; \Omega)
\end{gathered}
$$

with $a(\mathbf{v}, \mathbf{E})=\int_{\Omega}\left[\nabla \times \mathbf{v} \cdot \frac{1}{\mu_{r}} \nabla \times \mathbf{E}-k^{2} \mathbf{v} \cdot \epsilon_{r} \mathbf{E}\right] d V$ and $\langle\beta, \lambda\rangle_{\Gamma^{ \pm}}=\int_{\Gamma^{ \pm}}(\beta \cdot \lambda) d S$.

As for the exterior region $\Omega^{c}$, we start with the Stratton-Chu representation formula [5] 


$$
\mathbf{E}(\mathbf{x})=\Psi_{M}\left(\mathbf{e}^{+}\right)(\mathbf{x})-\Psi_{A}\left(\mathbf{j}^{+}\right)(\mathbf{x})-\frac{1}{k^{2}} \nabla \Psi_{V}\left(\nabla_{\Gamma} \cdot \mathbf{j}^{+}\right)(\mathbf{x}) \mathbf{x} \notin \Gamma
$$

Here $\Psi_{M}(\cdot), \Psi_{A}(\cdot)$, and $\Psi_{V}(\cdot)$ are potentials. $\Psi_{V}$ is the scalar single layer potential given by

$$
\Psi_{V}(\phi)(\mathbf{x})=\int_{\Gamma^{+}} G(\mathbf{x}, \mathbf{y}) \phi(\mathbf{y}) d S(\mathbf{y}) \quad \mathbf{x} \notin \Gamma
$$

with the Helmholtz kernel $G(\mathbf{x}, \mathbf{y})=\frac{\exp (i k|\mathbf{x}-\mathbf{y}|)}{4 \pi|\mathbf{x}-\mathbf{y}|}, \mathbf{x} \neq \mathbf{y} . \Psi_{A}$ is the vector version of the single layer potential; and, $\Psi_{M}$ is the vector double layer potential given by

$$
\Psi_{M}(\mathbf{v})(\mathbf{x})=\int_{\Gamma^{+}}\left(\nabla_{\mathbf{y}} G(\mathbf{x}, \mathbf{y}) \times \mathbf{v}\right) d S(\mathbf{y})
$$

The variational formulation for the surface traces, $\mathbf{e}^{+}$and $\mathbf{j}^{+}$, can be obtained using the exterior Calderon projector [5]. We write:

Seek $\mathbf{e}^{+} \in \mathbf{H}^{-1 / 2}\left(\operatorname{curl}_{\Gamma}, \Gamma^{+}\right)$and $\mathbf{j}^{+} \in \mathbf{H}^{-1 / 2}\left(\operatorname{div}_{\Gamma}, \Gamma^{+}\right)$such that

$$
\begin{gathered}
\left\langle\lambda^{+}, \mathbf{e}^{+}\right\rangle_{\Gamma^{+}}=\left\langle\lambda^{+},\left(\frac{1}{2} \mathcal{I}+\mathcal{C}\right)\left(\mathbf{e}^{+}\right)\right\rangle_{\Gamma^{+}}-\left\langle\lambda^{+}, \mathcal{S}\left(\mathbf{j}^{+}\right)\right\rangle_{\Gamma^{+}} \\
\left\langle\beta^{+}, \mathbf{j}^{+}\right\rangle_{\Gamma^{+}}=\left\langle\beta^{+}, \mathcal{N}\left(\mathbf{e}^{+}\right)\right\rangle_{\Gamma^{+}}+\left\langle\beta^{+},\left(\frac{1}{2} \mathcal{I}-\mathcal{B}\right)\left(\mathbf{j}^{+}\right)\right\rangle_{\Gamma^{+}} \\
\forall \beta^{+} \in \mathbf{H}^{-1 / 2}\left(\operatorname{curl}_{\Gamma}, \Gamma^{+}\right) \text {and } \lambda^{+} \in \mathbf{H}^{-1 / 2}\left(\operatorname{div}_{\Gamma}, \Gamma^{+}\right) .
\end{gathered}
$$

where the operators are:

$$
\begin{aligned}
\mathcal{S}:=\gamma_{t} \Psi_{S} & : \mathbf{H}^{-1 / 2}\left(\operatorname{div}_{\Gamma}, \Gamma\right) \mapsto \mathbf{H}^{-1 / 2}\left(\operatorname{curl}_{\Gamma}, \Gamma\right) \\
\mathcal{B}:=\frac{1}{2}\left(\gamma_{N}^{-}+\gamma_{N}^{+}\right) \Psi_{A} & : \mathbf{H}^{-1 / 2}\left(\operatorname{div}_{\Gamma}, \Gamma\right) \mapsto \mathbf{H}^{-1 / 2}\left(\operatorname{div}_{\Gamma}, \Gamma\right) \\
\mathcal{C}:=\frac{1}{2}\left(\gamma_{t}^{-}+\gamma_{t}^{+}\right) \Psi_{M} & : \mathbf{H}^{-1 / 2}\left(\operatorname{curl}_{\Gamma}, \Gamma\right) \mapsto \mathbf{H}^{-1 / 2}\left(\operatorname{curl}_{\Gamma}, \Gamma\right) \\
\mathcal{N}:=\gamma_{N} \Psi_{M} & : \mathbf{H}^{-1 / 2}\left(\operatorname{curl}_{\Gamma}, \Gamma\right) \mapsto \mathbf{H}^{-1 / 2}\left(\operatorname{div}_{\Gamma}, \Gamma\right)
\end{aligned}
$$

where $\Psi_{S}(\mathbf{j})=\Psi_{A}(\mathbf{j})+\frac{1}{k^{2}} \nabla \Psi_{V}\left(\nabla_{\Gamma} \cdot \mathbf{j}\right)$.

Moreover, the corresponding variational statement for the transmission conditions described in Eq. 5 is

$$
\begin{gathered}
\text { Seek }\left(\mathbf{e}^{-}, \mathbf{e}^{+}\right) \in \mathbf{H}^{-1 / 2}\left(\operatorname{curl}_{\Gamma}, \Gamma^{-}\right) \times \mathbf{H}^{-1 / 2}\left(\operatorname{curl}_{\Gamma}, \Gamma^{+}\right) \text {and } \\
\left(\mathbf{j}^{-}, \mathbf{j}^{+}\right) \in \mathbf{H}^{-1 / 2}\left(\operatorname{div}_{\Gamma}, \Gamma^{-}\right) \times \mathbf{H}^{-1 / 2}\left(\operatorname{div}_{\Gamma}, \Gamma^{+}\right) \text {such that } \\
\left\langle\lambda^{-}, \mathbf{e}^{-}\right\rangle_{\Gamma^{-}}+\frac{i}{k}\left\langle\lambda^{-}, \mathbf{j}^{-}\right\rangle_{\Gamma^{-}}=\left\langle\lambda^{-}, \mathbf{e}^{+}\right\rangle_{\Gamma^{-}}-\frac{i}{k}\left\langle\lambda^{-}, \mathbf{j}^{+}\right\rangle_{\Gamma^{-}}-\frac{i}{k}\left\langle\lambda^{-}, \mathbf{f}^{i n c}\right\rangle \Gamma^{-} \\
-i k\left\langle\beta^{-}, \mathbf{e}^{-}\right\rangle_{\Gamma^{-}}+\left\langle\beta^{-}, \mathbf{j}^{-}\right\rangle_{\Gamma^{-}}=-i k\left\langle\beta^{-}, \mathbf{e}^{+}\right\rangle_{\Gamma^{-}}-\left\langle\beta^{-}, \mathbf{j}^{+}\right\rangle_{\Gamma^{-}}-\left\langle\beta^{-}, \mathbf{f}^{i n c}\right\rangle_{\Gamma^{-}}
\end{gathered}
$$




$$
\begin{aligned}
\left\langle\lambda^{+}, \mathbf{e}^{+}\right\rangle_{\Gamma^{+}}+\frac{i}{k}\left\langle\lambda^{+}, \mathbf{j}^{+}\right\rangle_{\Gamma^{+}} & =\left\langle\lambda^{+}, \mathbf{e}^{-}\right\rangle_{\Gamma^{+}}-\frac{i}{k}\left\langle\lambda^{+}, \mathbf{j}^{-}\right\rangle_{\Gamma^{+}}+\frac{i}{k}\left\langle\lambda^{+}, \mathbf{g}^{i n c}\right\rangle_{\Gamma^{+}} \\
-i k\left\langle\beta^{+}, \mathbf{e}^{+}\right\rangle_{\Gamma^{+}}+\left\langle\beta^{+}, \mathbf{j}^{+}\right\rangle_{\Gamma^{+}} & =-i k\left\langle\beta^{+}, \mathbf{e}^{-}\right\rangle_{\Gamma^{+}}-\left\langle\beta^{+}, \mathbf{j}^{-}\right\rangle_{\Gamma^{+}}+\left\langle\beta^{+}, \mathbf{g}^{i n c}\right\rangle_{\Gamma^{+}}
\end{aligned}
$$

$$
\begin{gathered}
\forall\left(\beta^{-}, \beta^{+}\right) \in \mathbf{H}^{-1 / 2}\left(\operatorname{curl}_{\Gamma}, \Gamma^{-}\right) \times \mathbf{H}^{-1 / 2}\left(\operatorname{curl}_{\Gamma}, \Gamma^{+}\right) \text {and } \\
\left(\lambda^{-}, \lambda^{+}\right) \in \mathbf{H}^{-1 / 2}\left(\operatorname{div}_{\Gamma}, \Gamma^{-}\right) \times \mathbf{H}^{-1 / 2}\left(\operatorname{div}_{\Gamma}, \Gamma^{+}\right)
\end{gathered}
$$

Substituting Eq. 10 into Eq. 14 results in

$$
\begin{aligned}
&\left\langle\lambda^{+},\left(\frac{1}{2} \mathcal{I}+\mathcal{C}\right)\left(\mathbf{e}^{+}\right)\right\rangle_{\Gamma^{+}}-\left\langle\lambda^{+}, \mathcal{S}\left(\mathbf{j}^{+}\right)\right\rangle_{\Gamma^{+}}+\frac{i}{k}\left\langle\lambda^{+}, \mathbf{j}^{+}\right\rangle_{\Gamma^{+}} \\
&=\left\langle\lambda^{+}, \mathbf{e}^{-}\right\rangle_{\Gamma^{+}}-\frac{i}{k}\left\langle\lambda^{+}, \mathbf{j}^{-}\right\rangle_{\Gamma^{+}}+\frac{i}{k}\left\langle\lambda^{+}, \mathbf{g}^{i n c}\right\rangle_{\Gamma^{+}} \\
&-i k\left\langle\beta^{+}, \mathbf{e}^{+}\right\rangle_{\Gamma^{+}}+\left\langle\beta^{+}, \mathcal{N}\left(\mathbf{e}^{+}\right)\right\rangle_{\Gamma^{+}}+\left\langle\beta^{+},\left(\frac{1}{2} \mathcal{I}-\mathcal{B}\right)\left(\mathbf{j}^{+}\right)\right\rangle_{\Gamma^{+}} \\
&=-i k\left\langle\beta^{+}, \mathbf{e}^{-}\right\rangle_{\Gamma^{+}}-\left\langle\beta^{+}, \mathbf{j}^{-}\right\rangle_{\Gamma^{+}}+\left\langle\beta^{+}, \mathbf{g}^{i n c}\right\rangle_{\Gamma^{+}}
\end{aligned}
$$

Finally, we state the overall variational formulation for the proposed nonconformal coupling between finite and boundary elements:

Seek $\mathbf{E} \in \mathbf{H}(\operatorname{curl} ; \Omega), \mathbf{j}^{-} \in \mathbf{H}^{-1 / 2}\left(\operatorname{div}_{\Gamma}, \Gamma^{-}\right), \mathbf{e}^{+} \in \mathbf{H}^{-1 / 2}\left(\operatorname{curl}_{\Gamma}, \Gamma^{+}\right)$, and $\mathbf{j}^{+} \in \mathbf{H}^{-1 / 2}\left(\operatorname{div}_{\Gamma}, \Gamma^{+}\right)$such that

$a(\mathbf{v}, \mathbf{E})-\frac{1}{2}\left\langle\gamma_{t} \mathbf{v}, \mathbf{j}^{-}\right\rangle_{\Gamma^{-}}-\frac{i k}{2}\left\langle\gamma_{t} \mathbf{v}, \mathbf{e}^{-}\right\rangle_{\Gamma^{-}}+\frac{i k}{2}\left\langle\gamma_{t} \mathbf{v}, \mathbf{e}^{+}\right\rangle_{\Gamma^{-}}+\frac{1}{2}\left\langle\gamma_{t} \mathbf{v}, \mathbf{j}^{+}\right\rangle_{\Gamma^{-}}$ $=-\frac{1}{2}\left\langle\gamma_{t} \mathbf{v}, \mathbf{f}^{i n c}\right\rangle_{\Gamma^{-}}$

$-\frac{1}{2}\left\langle\lambda^{-}, \mathbf{e}^{-}\right\rangle_{\Gamma^{-}}-\frac{i}{2 k}\left\langle\lambda^{-}, \mathbf{j}^{-}\right\rangle_{\Gamma^{-}}+\frac{1}{2}\left\langle\lambda^{+}, \mathbf{e}^{+}\right\rangle_{\Gamma^{-}}-\frac{i}{2 k}\left\langle\lambda^{-}, \mathbf{j}^{+}\right\rangle_{\Gamma^{-}}$

$=\frac{i}{2 k}\left\langle\lambda^{-}, \mathbf{f}^{i n c}\right\rangle_{\Gamma^{-}}$

$-\frac{i k}{2}\left\langle\beta^{+}, \mathbf{e}^{+}\right\rangle_{\Gamma^{+}}+\frac{1}{2}\left\langle\beta^{+}, \mathcal{N}\left(\mathbf{e}^{+}\right)\right\rangle_{\Gamma^{+}}+\frac{1}{2}\left\langle\beta^{+},\left(\frac{1}{2} \mathcal{I}-\mathcal{B}\right)\left(\mathbf{j}^{+}\right)\right\rangle_{\Gamma^{+}}$

$+\frac{i k}{2}\left\langle\beta^{+}, \mathbf{e}^{-}\right\rangle_{\Gamma^{+}}+\frac{1}{2}\left\langle\beta^{+}, \mathbf{j}^{-}\right\rangle_{\Gamma^{+}}=\frac{1}{2}\left\langle\beta^{+}, \mathbf{g}^{i n c}\right\rangle_{\Gamma^{+}}$

$-\frac{1}{2}\left\langle\lambda^{+},\left(\frac{1}{2} \mathcal{I}+\mathcal{C}\right)\left(\mathbf{e}^{+}\right)\right\rangle_{\Gamma^{+}}+\frac{1}{2}\left\langle\lambda^{+}, \mathcal{S}\left(\mathbf{j}^{+}\right)\right\rangle_{\Gamma^{+}}-\frac{i}{2 k}\left\langle\lambda^{+}, \mathbf{j}^{+}\right\rangle_{\Gamma^{+}}$

$-\frac{i}{2 k}\left\langle\lambda^{+}, \mathbf{j}^{-}\right\rangle_{\Gamma^{+}}+\frac{1}{2}\left\langle\lambda^{+}, \mathbf{e}^{-}\right\rangle_{\Gamma^{+}}=-\frac{i}{2 k}\left\langle\lambda^{+}, \mathbf{g}^{i n c}\right\rangle_{\Gamma^{+}}$

$\forall \mathbf{v} \in \mathbf{H}(\mathbf{c u r l} ; \Omega), \lambda^{-} \in \mathbf{H}^{-1 / 2}\left(\operatorname{div}_{\Gamma}, \Gamma^{-}\right), \beta^{+} \in \mathbf{H}^{-1 / 2}\left(\operatorname{curl}_{\Gamma}, \Gamma^{+}\right)$, and $\lambda^{+} \in \mathbf{H}^{-1 / 2}\left(\operatorname{div}_{\Gamma}, \Gamma^{+}\right)$. 


\subsection{Matrix Equation for the Nonconformal Coupling Between Finite and Boundary Elements}

In the finite dimensional discretization, we have employed the following approximations in tetrahedra and on triangles for the variables:

$$
\begin{aligned}
& \mathbf{E}: \text { second order Nédélec elements of the } 1^{\text {st }} \text { kind [11] in } \Omega_{h} \\
& \mathbf{e}^{-}: \gamma_{t} \mathbf{E} \text { on } \Gamma_{h}^{-} \\
& \mathbf{j}^{-}: \text {second order Raviart-Thomas elements [12] on } \Gamma_{h}^{-} \\
& \mathbf{e}^{+}: \text {edge elements on } \Gamma_{h}^{+} \\
& \mathbf{j}^{+}: \text {first order Raviart-Thomas elements [12] on } \Gamma_{h}^{+}
\end{aligned}
$$

Subsequently, the final matrix equation corresponds to the variational formulation 15 is of the form

$$
\left[\begin{array}{ccccc}
A_{I I} & A_{I \Gamma} & 0 & 0 & 0 \\
A_{\Gamma I} & A_{\Gamma \Gamma}-\frac{i k}{2} T_{\Gamma^{-} \Gamma^{-}} & \frac{1}{2} D_{\Gamma^{-} \Gamma^{-}} & \frac{i k}{2} T_{\Gamma^{-} \Gamma^{-}} & \frac{1}{2} D_{\Gamma^{-} \Gamma^{+}} \\
0 & -\frac{1}{2} D_{\Gamma^{-} \Gamma^{-}}^{t} & \frac{i}{2 k} T_{\Gamma^{-} \Gamma^{-}} & \frac{1}{2} D_{\Gamma^{-} \Gamma^{+}}^{t} & -\frac{i}{2 k} T_{\Gamma^{-} \Gamma^{+}} \\
0 & \frac{i k}{2} T_{\Gamma^{-} \Gamma^{+}}^{t} & \frac{1}{2} D_{\Gamma^{-} \Gamma^{+}}^{t} & \frac{1}{2} Q_{e}-\frac{i k}{2} T_{\Gamma^{+} \Gamma^{+}} & \frac{1}{2} P \\
0 & \frac{1}{2} D_{\Gamma^{-} \Gamma^{+}}^{t} & -\frac{i}{2 k} T_{\Gamma^{-} \Gamma^{+}}^{t} & \frac{1}{2} U\left(\equiv P^{t}\right) & \frac{1}{2} Q_{j}-\frac{i}{2 k} T_{\Gamma^{+} \Gamma^{+}}
\end{array}\right]\left[\begin{array}{c}
\mathbf{E}_{\text {int }} \\
\mathbf{e}^{-} \\
\mathbf{j}^{-} \\
\mathbf{e}^{+} \\
\mathbf{j}^{+}
\end{array}\right]
$$

Note that in Eq. 16, we have partitioned the unknown coefficients of $\mathbf{E}$ into $\mathbf{E}_{\text {int }}$ and $\mathbf{e}^{-}$for the interior and surface unknowns, respectively. The submatrices and their corresponding bilinear forms are summarized below

$$
\begin{array}{ccc}
{\left[\begin{array}{cc}
A_{I I} & A_{I \Gamma} \\
A_{\Gamma I} & A_{\Gamma \Gamma}
\end{array}\right]: a(\mathbf{v}, \mathbf{E})} & T_{\Gamma^{-} \Gamma^{-}}:\left\langle\gamma_{t} \mathbf{v}, \mathbf{e}^{-}\right\rangle_{\Gamma^{-}} & T_{\Gamma^{+} \Gamma^{+}}:\left\langle\beta^{+}, \mathbf{e}^{+}\right\rangle_{\Gamma^{+}} \\
T_{\Gamma^{-} \Gamma^{+}}:\left\langle\gamma_{t} \mathbf{v}, \mathbf{e}^{+}\right\rangle_{\Gamma^{-}} & D_{\Gamma^{-} \Gamma^{-}}:\left\langle\gamma_{t} \mathbf{v}, \mathbf{j}^{-}\right\rangle_{\Gamma^{-}} & D_{\Gamma^{-} \Gamma^{+}}:\left\langle\gamma_{t} \mathbf{v}, \mathbf{j}^{+}\right\rangle_{\Gamma^{-}} \\
Q_{e}:\left\langle\beta^{+}, \mathcal{N}\left(\mathbf{e}^{+}\right)\right\rangle_{\Gamma^{+}} & Q_{j}:\left\langle\lambda^{+}, \mathcal{S}\left(\mathbf{j}^{+}\right)\right\rangle_{\Gamma^{+}} & P:\left\langle\beta^{+},\left(\frac{1}{2} \mathcal{I}-\mathcal{B}\right)\left(\mathbf{j}^{+}\right)\right\rangle_{\Gamma^{+}} \\
U:\left\langle\lambda^{+},\left(\frac{1}{2} \mathcal{I}+\mathcal{C}\right)\left(\mathbf{e}^{+}\right)\right\rangle_{\Gamma^{+}} & &
\end{array}
$$

\section{Numerical Results}

In Figure 1, we show the condition numbers of the final matrix equations resulted from the symmetric couplings based on the Costabel approach $[6,5]$ and the new proposed non-conformal coupling for a box computational domain. 
Note that Figure 1(a) and (b) clear indicate that the previous symmetric formulations suffer the notorious internal resonances, whereas the new proposed approach does not. Moreover, in Figure 1(c), we plot the eigenvalues distribution of the same matrix (from the proposed method) of the off-diagonal blocks after applying the block diagonal preconditioner [8]. All the eigenvalues are within the unit circle, and clearly observe the C.B.S. inequality. In Figure 2, the bistatic radar cross section (RCS) computed using the proposed method for a metallic generic battle ship are compared with those obtained by a fast boundary element code, based on electric field integral equation (EFIE). The agreement is excellent between the two results and hence validate the accuracy of the proposed approach.

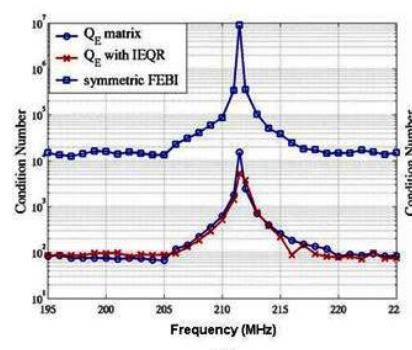

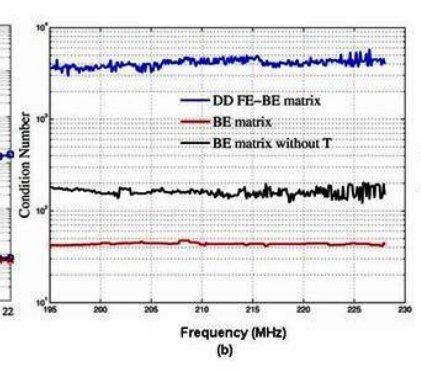

(b)

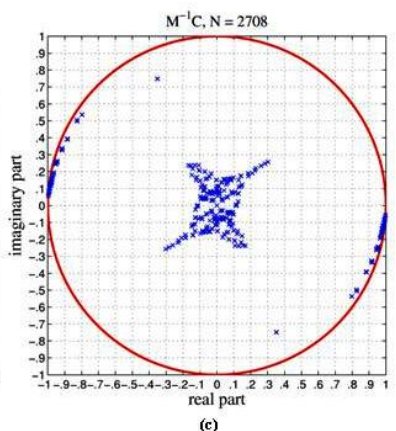

Fig. 1. Condition numbers and eigenvalue distributions of the coupled finite elements and boundary elements formulations for a box domain. (a) The symmetric formulation based on Costabel approach [6, 5]; (b) The currently proposed approach; and, (c) Eigenvalues distribution of the off-diagonal blocks after preconditioned. Note that all the eigenvalues are within the unit circle and thus satisfied the C.B.S inequality [8].

\section{Conclusions}

This chapter describes a variational formulation for non-conformal couplings between finite and boundary elements for electromagnetic scattering problems in $\mathcal{R}^{3}$. Numerical examples demonstrate the proposed DD-FE-BE formulation does not suffer the notorious internal resonances and results in matrix equations that satisfy the C.B.S. inequality after applying the block diagonal preconditioner.

\section{References}

1. J.-M. Jin, J. L. Volakis, and J. D. Collins. A finite-element-boundary integral method for scattering and radiation by two and three-dimensional structures. IEEE Antennas and Propagation Magazine, 33(3):22-32, 1991. 


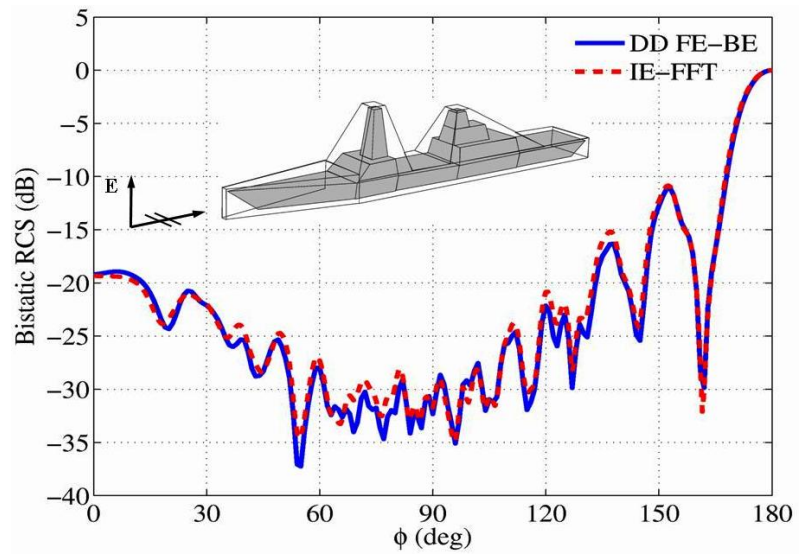

Fig. 2. Comparisons of the computed bi-static RCS using the proposed DD-FE-BE method and the IE-FFT accelerated boundary element method.

2. J. Liu and J.-M. Jin. A novel hybridization of higher order finite element and boundary integral methods for electromagnetic scattering and radiation problems. IEEE Transactions on Antennas and Propagation, 49(12):1794-1806, 2001.

3. C. Brebbia, W. Wendland, and G. Kuhn, editors. Boundary Elements IX, chapter M. Costabel, "Symmetric methods for the coupling of finite elements and boundary elements", pages 411-420. Springer-Verlag, 1987.

4. R. Hiptmair. Symmetric coupling for eddy current problems. SIAM J. Numer. Anal., 40(1):41-65, 2002.

5. R. Hiptmair. Coupling of finite elements and boundary elements in electromagnetic scattering. SIAM J. Numer. Anal., 41(3):919-944, 2003.

6. M. N. Vouvakis, S.-C. Lee, K. Zhao, and J.-F. Lee. A symmetric fem-ie formulation with a single-level ie-qr algorithm for solving electromagnetic radiation and scattering problems. IEEE Trans. Antennas and Propagation, 52(11):30603070, Nov. 2004.

7. S.-C. Lee, M. N. Vouvakis, K. Zhao, and J.-F. Lee. Analyzing microwave devices using a symmetric coupling of finite and boundary elements. International Journal for Numerical Methods in Engineering, To Appear, 2005.

8. O. Axelsson. Iterative SOlution Methods. Cambridge University Press, $1^{\text {st }}$ edition, 1996.

9. S.-C. Lee, M. Vouvakis, and J.-F. Lee. A non-overlapping domain decomposition method with non-matching grids for modeling large finite antenna arrays. Journal of Comput. Phys., 203:1-21, Feb. 2005.

10. D. K. Sun, Z. J. Cendes, and J.-F. Lee. Adaptive mesh refinement, h-version, for solving multiport microwave devices in three dimensions. IEEE Trans. Magn, 36(4):1596-1599, July 2000.

11. J.-C. Nédélec. Mixed finite elements in $\mathcal{R}^{3}$. Numer. Math., 35:315-341, 1980.

12. A. Dold and B. Eckmann, editors. Mathmatical Aspects of Finite Element Methods, volume 606 of Lecture Notes of Mathematics, chapter P.-A. Raviart and J.M. Thomas, "A Mixed Finite Element Method for $2^{\text {nd }}$ Order Elliptic Problems". Springer, 1975. 